\title{
Synthesis of fully-fused bisboron azomethine complexes and their conjugated polymers with solid- state near-infrared emission
}

\section{$\operatorname{AUTHOR}(S):$}

Ohtani, Shunsuke; Nakamura, Masashi; Gon, Masayuki; Tanaka, Kazuo; Chujo, Yoshiki

\section{CITATION:}

Ohtani, Shunsuke ... [et al]. Synthesis of fully-fused bisboron azomethine complexes and their conjugated polymers with solid-state near-infrared emission. Chemical

Communications 2020, 56(48): 6575-6578

\section{ISSUE DATE:}

2020-06-18

URL:

http://hdl.handle.net/2433/254052

\section{RIGHT:}

This is the accepted manuscript of the article, which has been published in final form at

https://doi.org/10.1039/d0cc02301a.; The full-text file will be made open to the public on $18 \mathrm{June} 2021$ in accordance with publisher's 'Terms and Conditions for Self-Archiving'.; この論文は出版社版でありません。引用の際には出版社版 をご確認ご利用ください。; This is not the published version. Please cite only the published version. 


\section{COMMUNICATION}

Received 00th January 20xx, Accepted 00th January 20xx DOI: $10.1039 / \times 0 \times x 00000 x$

\section{Synthesis of Fully-Fused Bisboron Azomethine Complexes and Their Conjugated Polymers with Solid-State Near-Infrared Emission}

\author{
Shunsuke Ohtani, Masashi Nakamura, Masayuki Gon, Kazuo Tanaka* and Yoshiki Chujo
}

We describe here the elemental skeleton for realizing robust $\pi$ conjugated molecules with solid-state emission in the nearinfrared (NIR) region $\left(\Phi_{\mathrm{F}}=0.03-0.06\right)$. Initially, the diastereomers of bisboron azomethine complexes having phenyl groups with the same and opposite directions toward $\pi$-plane were synthesized. These diastereomers showed emission properties with larger redshifts (>200 $\mathbf{n m})$ and $\mathbf{1 0}$ times larger emission efficiencies than the mononuclear complex. Theoretical calculation data indicate that superior optical properties of the bisboron complexation should be attributable to efficient expansion of $\pi$-conjugated system. In addition, the bisboron compounds and their conjugated polymers exhibited intense NIR emissions even in the solid states.

Near-infrared (NIR) light has various advantageous features such as low photodamage to biological samples, deep tissue penetration and minimum interference from background autofluorescence by biomolecules in the living systems. ${ }^{1}$ In addition, if dyes have solid-state luminescent properties, which are mostly spoiled in conventional luminescent dyes, these dyes can be a key unit in advanced optical devices, such as telecommunication devices ${ }^{2}$, laser amplifiers ${ }^{3}$ and organic lightemitting diodes (OLEDs) ${ }^{4}$. One of the strategies for obtaining NIR-emissive dyes is to reduce the energy band gap $\left(E_{\mathrm{g}}\right)$ between HOMO (highest occupied molecular orbital) and LUMO (lowest unoccupied molecular orbital) levels by using extended $\pi$-conjugated systems. ${ }^{5}$ As is often the case with polymer syntheses, another strategy is to construct an alternating array of electron-donor and acceptor moieties. Because HOMO and LUMO levels can be elevated and lowered by electron donors and acceptors, respectively, intense emission in longer wavelength regions can be obtained. ${ }^{6}$ Although there are these established design strategies, there are still a limited number of emissive organic dyes having NIR and especially solid-state luminescent properties. In solid states,

Department of Polymer Chemistry, Graduate School of Engineering, Kyoto University Katsura, Nishikyo-ku, Kyoto 615-8510, Japan. E-mail: tanaka@poly.synchem.kyoto-u.ac.jp

†Electronic Supplementary Information (ESI) available. See

DOI: 10.1039/x0xx00000x organic chromophores having large $\pi$-conjugated systems facilitate the consecutive $\pi-\pi$ stacking and other electronic interactions (e.g., energy transfer, inter-, or intramolecular charge transfer, and excited states reactions). ${ }^{7}$ As a result, these materials have been suffering from the aggregation caused quenching (ACQ) effect. ${ }^{8}$

Recently, we proposed the concept of "element-blocks", which are minimum functional units containing heteroatoms, to create functional materials according to preprogrammed designs. ${ }^{9}$ On the basis of this strategy, it has been revealed that introduction of heteroatoms into organic scaffolds is one of the facile and effective methods to expand $\pi$-conjugated systems. In particular, boron-containing "element-blocks" are promising building blocks for developing luminescent materials by constructing planar and rigid conjugated systems. ${ }^{10}$ Furthermore, ladder-type $\pi$-conjugated multi-boron complexes, possessing fused polycyclic skeletons with four coordinated boron atoms, have ideal emissive properties since their effectively-extended $\pi$-conjugated systems can contribute to the formation of the planar and rigid molecular skeletons. ${ }^{11}$ In the recent report, we reported synthesis and unique solid-state luminescent properties of the boron-fused azomethine complex modified with a diethylamino group. It was observed that this complex is able to avoid ACQ and shows intense emission in crystal larger than those in amorphous and diluted solution

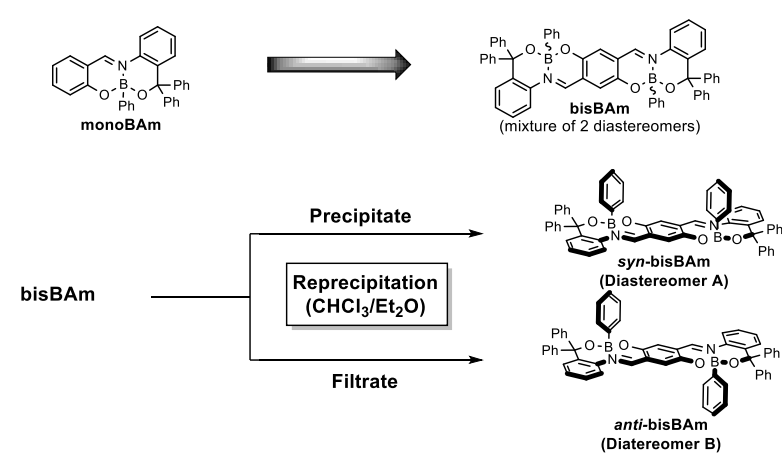

Fig 1. Chemical structures of svn-. anti-bisBAm and monoBAm. 
state (crystallization-induced emission enhancement, CIEE). ${ }^{12,13}$ Furthermore, the donor-acceptor conjugated copolymers involving the boron-fused azomethine acceptor unit exhibited intense emission even in the thin films. ${ }^{14}$ These results suggest that $\pi$-conjugated systems should be extensively developed through the boron-fused azomethine unit. Particularly, the azomethine unit could be responsible for suppressing ACQ followed by induction of solid-state luminescence despite that boron-fused structures have highly-planar structures which are favorable for extending conjugated systems, while ACQ often occurs due to non-specific intermolecular interactions. From these results, we presumed that further extension of $\pi$ conjugated systems involving the boron-fused azomethine complexes might be a potential design for obtaining a solidstate emissive material in the NIR region.

Herein, we report the bisboron complexes having the phenyl capping at the boron atoms composed of the fused structure which can contribute to construction of robust $\pi$-conjugated system based on the azomethine scaffold. Two kinds of diastereomers were successfully isolated, and it was observed that the bisboron complexes exhibited NIR emission not only in solution but also in solid because of steric hindrances of the phenyl substituents. Additionally, owing to good solubility in organic solvents, polymerization was accomplished utilizing the complex as a monomer. From the alternating copolymers, large degrees of red-shifted absorption and emission spectra were observed compared to those of bisboron complexes as well as mono-boron ones, indicating that $\pi$-conjugated system should be efficiently developed through whole molecules. Finally, NIRluminescent film materials can be obtained with the polymers through an ordinal spin-coating method.

The bisboron complex bisBAm containing two boron-fused structures was synthesized from the azomethine tridentate ligand 3 (Schemes S1-S3 and Fig. 1 and S1-S9). To evaluate the effect on the expansion of $\pi$-conjugated system by the bisboron complexation, we also synthesized the mononuclear boron complex monoBAm (Schemes S4-S5 and Fig. 1 and S10-S14). ${ }^{1} \mathrm{H}$ NMR spectrum of bisBAm showed two singlet peaks at $\delta=$ $8.04,8.05 \mathrm{ppm}$ which corresponded to the azomethine protons, indicating that bisBAm is obtained as a diastereomeric mixture (syn- and anti-bisBAm) due to the stereogenic boron centers (Fig. S28). To examine the optical properties of these diastereomers individually, we tried separating these diastereomers by a reprecipitation method. After pouring $\mathrm{Et}_{2} \mathrm{O}$ (poor solvent) to the diastereomeric mixture solution in $\mathrm{CHCl}_{3}$ (good solvent) and stirring for $1 \mathrm{~h}$ at room temperature, the precipitation was generated. After filtration, the solvent was removed from the filtrate to afford diastereomer $A$ as a deep blue powder. We also successfully obtained diastereomer B as a deep blue powder by purifying the precipitate. Fortunately, single crystal pieces of diastereomer B with sufficient size for a single-crystal $\mathrm{X}$-ray analysis were obtained by a vapor diffusion method from the $\mathrm{CHCl}_{3}$ solution under $\mathrm{Et}_{2} \mathrm{O}$ atmosphere. According to the data at $106 \mathrm{~K}$, diastereomer $\mathrm{B}$ was assigned to anti-bisBAm in which the two phenyl moieties protruded from the four-coordinated boron atoms were opposite side of the molecular framework (Fig. 2 and Table S1). There seems to be

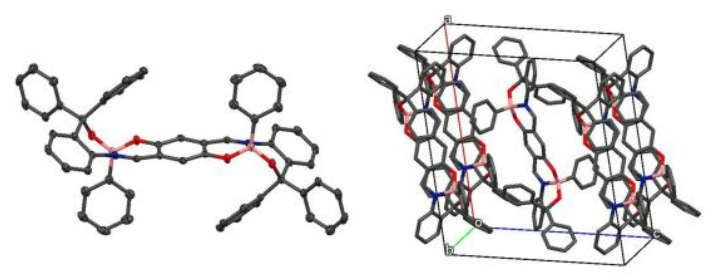

Fig. 2 Thermal ellipsoid (probability level 50\%) (left) and packing structure (right) of anti-bisBAm Hydrogen atoms were omitted for clarity.

subtle interfacial $\pi-\pi$ interaction in the crystal packing of antibisBAm because large degree of steric hindrance of the six phenyl groups prevented the overlap among the $\pi$-conjugated skeletons. From these results, the remaining diastereomer $A$ was assigned as syn-bisBAm where phenyl groups on boron atoms were the same side of the molecular plane.

In UV-vis absorption spectra (Fig. 3a) of bisboron complexes syn- and anti-bisBAm in $\mathrm{CHCl}_{3}$ diluted solution, the longest absorption bands showed remarkable red-shift from that of monoBAm (Table 1). This result suggested that the conjugated systems in the ground state should be efficiently extended by bisboron complexation. Additionally, from the TD-DFT (timedependent density functional theory) calculations at the B3LYP/6-311G(d,p) level (See Supporting Information), it was shown that the molecular orbitals of both HOMO and LUMO were more delocalized over the entire molecules in syn- and anti-bisBAm, compared to that of monoBAm (Fig. S34). Corresponded to the molecular orbitals spread, the $E_{\mathrm{g}}$ between the HOMO and LUMO of syn- and anti-bisBAm became narrower than that of monoBAm. The energy levels were determined by the calculation with the optical band gap ( $\left.\Delta E_{\mathrm{g}, \mathrm{opt}}\right)$ obtained from the UV-vis absorption spectra $\left(E_{\text {Hомо }}=E_{\mathrm{LUMO}}-\right.$ $\Delta E_{\mathrm{g}, \mathrm{opt}}$ ) and the data from cyclic voltammetry (CV) (Fig. S36). Accordingly, bisboron complexes showed narrower HOMOLUMO gaps than that of monoBAm originating from increases and decreases in HOMO and LUMO levels respectively. It should be emphasized that the decreases in LUMO levels were much larger than the increases in HOMO levels, suggesting that the electron-accepting ability should be enhanced by introducing the second boron-fused moieties.

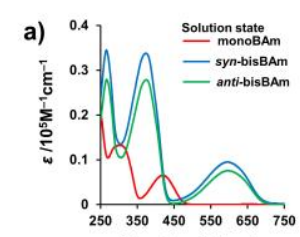

c) 0. Wavelength $(\mathrm{nm})$

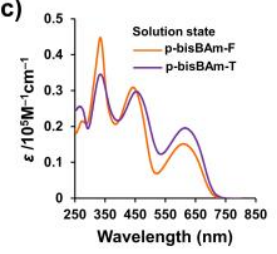

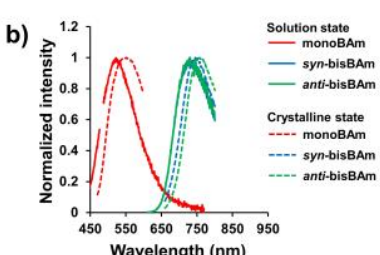

d)

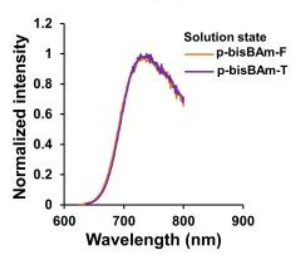

Fig. 3 a, c) UV-vis absorption and b, d) photoluminescence spectra of monoBAm, syn-bisBAm, anti-bisBAm, p-bisBAm- $\mathbf{F}$ and p-bisBAm- $\mathbf{T}$ in the solution $\left(\mathrm{CHCl}_{3}\right.$, $\left.1.0 \times 10^{-5} \mathrm{M}\right)$ and crystalline states. 
Table 1 Optical properties of synthesized compounds in solution state ${ }^{a}$

\begin{tabular}{ccccccc}
\hline & $\begin{array}{c}\lambda_{\text {max,abs }} \\
(\mathrm{nm})^{b}\end{array}$ & $\begin{array}{c}\lambda_{\mathrm{em}} \\
(\mathrm{nm})^{c}\end{array}$ & $\Phi_{\mathrm{F}}{ }^{d}$ & $\begin{array}{c}\tau \\
(\mathrm{ns})^{e}\end{array}$ & $\begin{array}{c}k_{\mathrm{r}} \\
\left(\times 10^{8}\right. \\
\left.\mathrm{s}^{-1}\right)^{f}\end{array}$ & $\begin{array}{c}k_{\mathrm{nr}} \\
\left(\times 10^{8}\right. \\
\left.\mathrm{s}^{-1}\right)^{f}\end{array}$ \\
\hline monoBAm & 419 & 522 & 0.003 & - & - & - \\
syn-bisBAm & 599 & 731 & 0.035 & 1.5 & 0.23 & 6.2 \\
anti-bisBAm & 595 & 729 & 0.038 & 1.5 & 0.25 & 6.3 \\
p-bisBAm-F & 610 & 742 & 0.060 & 1.4 & 0.47 & 6.6 \\
p-bisBAm-T & 614 & 742 & 0.062 & 1.3 & 0.50 & 7.3 \\
\hline
\end{tabular}

${ }^{a} \mathrm{CHCl}_{3}$ solution, $1.0 \times 10^{-5} \mathrm{M}$ (per repeating units in the case of polymer samples). ${ }^{b}$ The longest absorption maximum. ${ }^{c}$ Fluorescence maxima with the excitation at the longest absorption maximum. ${ }^{d}$ Absolute fluorescence quantum yield. ${ }^{e}$ Emission lifetime at $\lambda_{\mathrm{em}} \cdot{ }^{f} k_{\mathrm{r}}=\Phi_{\mathrm{F}} / \tau, k_{\mathrm{nr}}=\left(1-\Phi_{\mathrm{F}}\right) / \tau$

Photoluminescence (PL) spectra of syn- and anti-bisBAm shows dramatically red-shifted emission bands by more than $200 \mathrm{~nm}$ compared to that of monoBAm and their emission maxima reached to the NIR region (Fig. $3 \mathrm{~b}$ and S31). These exceptionally-large degree of red-shifts comparing to the corresponding mono-nuclear boron complex have never been reported in previous literatures regarding $\pi$-conjugated multiboron compounds. ${ }^{11 f, h, j, k}$ This NIR luminescent properties should be originated from the robust conjugation involving the linearly-connected tandem azomethine units assisted by the fully-fused borons for realizing a planar and rigid molecular framework. Furthermore, their absolute fluorescence quantum yields ( $\Phi_{\mathrm{F}} \mathrm{S}$ ) were about 10 times higher than that of monoBAm. To investigate influence of molecular motions on luminescent properties, emission yields were monitored with polystyrene films including $1 \mathrm{wt} \%$ of monoBAm or bisBAm (Table S3, Fig. S29 and S32). The $\Phi_{\mathrm{F}}$ of monoBAm in the film state increased to be 0.013 . In addition, the smaller Stokes shifts were observed in the film than that in the solution state, clearly indicating that the excited state of monoBAm should be deactivated via nonradiative process in solution, and the decay process is able to be disturbed by the restriction of the intramolecular motion (Table S2-S3). Conversely, in the case of bisBAm, the $\Phi_{\mathrm{F}}$ value in the film state was hardly changed compared to that in the solution state, suggesting that bisboron complexation enhances rigidity into the molecular framework.

To get further insight about the kinetics, the radiative $\left(k_{\mathrm{r}}\right)$ and non-radiative rate constants $\left(k_{\mathrm{nr}}\right)$ were determined from a PL lifetime measurements under various conditions (Table 1 and Fig. S33). In the dispersed films of monoBAm and bisBAm compounds, it was found that the $k_{\mathrm{r}}$ of monoBAm was much smaller than those of bisBAm compounds, suggesting that the enhancement of $\Phi_{\mathrm{F}}$ by bisboron complexation should be originated from the large oscillator strength of $S_{1} \rightarrow S_{0}$ transition owing to the extension of the $\pi$-conjugated system. There was less significant difference between syn- and anti-bisBAm in the absorption and PL properties of the solution states. This fact suggests that the electronic structures of isolated bisBAm molecules are almost identical.

Next, the emissive properties of syn- and anti-bisBAm in crystalline states were examined (Table S4 and Fig. 3b). The NIR emissions of bisBAm can be detected even in the solid states with $\Phi_{\mathrm{F}}$ values (syn-bisBAm: $\Phi_{\mathrm{F}}=0.035$; anti-bisBAm: $\Phi_{\mathrm{F}}=$ 0.052 ). These values are relatively high for NIR emitters because, in general, the $\Phi_{\mathrm{F}}$ in the NIR region is significantly lower than that in the visible region due to the energy-gap law. In general, consecutive $\pi-\pi$ stacking leads to emission quenching in solid through migration of excited energy. On the other hand, the significant emission quenching was hardly observed in both synbisBAm and anti-bisBAm crystalline samples. This is probably because the protruded phenyl groups on boron atoms prevented $\pi-\pi$ stacking in crystal. As a result, the NIR emissive property in solution can be preserved even in crystal. Notably, it was found that the PL spectra of syn-bisBAm was observed in the longer wavelength region than that of anti-bisBAm (synbisBAm-F: $\lambda_{\mathrm{em}}=757 \mathrm{~nm}$; anti-bisBAm: $\lambda_{\mathrm{em}}=745 \mathrm{~nm}$ ) despite that the almost same emission properties were obtained in solution and the films. It should be because syn-bisBAm tends to form stronger intermolecular interaction between adjacent molecules than anti-bisBAm. This result might be responsible for the structural feature of syn-bisBAm, such as, a vacant molecular surface resulted from the phenyl groups on boron atoms orienting in the same direction.

To probe the applicability of bisboron complexes as a monomer for constructing conjugated polymers, we acturally synthesized the donor-acceptor type conjugated copolymers $\mathbf{p}$ bisBAm-F and p-bisBAm-T from the dibrominated bisBAmBr and fluorene, bithiophene comonomers (Schemes S6-S9, Fig. 4 and S15-S27). At this time, the monomer bisBAmBr was used as a diastereomeric mixture for polymerization due to difficulty in the separation of diastereomers. Polymerizations were accomplished by the palladium-catalyzed Migita-Kosugi-Stille coupling reaction ${ }^{15}$ with fluorene $(\mathbf{F})$ or bithiophene derivatives $(T)$ in toluene in the presence of conventional coupling reagents. The size-exclusion chromatography using $\mathrm{CHCl}_{3}$ as an eluent with the polystyrene standards clearly indicated generation of polymerization products (Table S6) In absorption spectra with the polymer solutions, the absorption bands were observed in much longer wavelength region with larger molar extinction coefficients than those of the monomeric bisBAm diastereomers, clearly proving that robust conjugation should be extended through polymer main chains (Table S2 and Fig. 3c). From TD-DFT calculations with model compounds $\mathbf{p}$-bisBAm- $\mathbf{F}^{\prime}$ and p-bisBAm-T', delocalization of HOMOs were observed, suggesting that extension of conjugated systems through polymer main chains plays a significant role in narrowing HOMO-LUMO energy band gaps and improving the oscillator strengths of $\mathrm{S}_{0} \rightarrow \mathrm{S}_{1}$ transition (Fig. S35). Although the HOMO

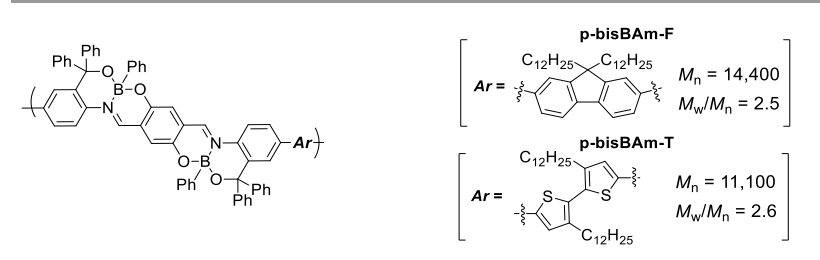

Fig 4. Chemical structures of $\mathbf{p}$-bisBAm-F and p-bisBAm-T.

orbitals of $\mathbf{p}$-bisBAm-F' and $\mathbf{p}$-bisBAm-T' were more delocalized 
than bisBAm, there was hardly significant difference between HOMO levels of fluorene and bithiophene. This theoretical result was consistent with experimentally obtained results from the optical measurements. We assume that the delocalization of the HOMO orbitals might be mostly limited within bisBAm scaffolds. As a result, the fluorene and bithiophene moieties did not influence the HOMO energy levels. Furthermore, the $\Phi_{\mathrm{F}} \mathrm{S}$ of p-bisBAm-F, -T were determined as 0.066 and 0.064 , respectively, which were almost twice higher than those of monomeric bisBAm compounds (Table 2 and Fig. 3d). According to the kinetic data (Table S2), larger $k_{\mathrm{r}} \mathrm{s}$ were observed from the polymers. This result supports that the fluorescence enhancement should be ascribed to the acceleration of radiation processes caused by extended $\pi$-conjugation. Finally, it was demonstrated that further red-shifted emission bands were obtained from the film samples without critical losses of $\Phi_{\mathrm{F}} \mathrm{S}$ (p-bisBAm-F: $\Phi_{\mathrm{F}}=0.049, \lambda_{\mathrm{em}}=751 \mathrm{~nm} ; \mathbf{p}$-bisBAm-T: $\Phi_{\mathrm{F}}=$ $0.042, \lambda_{\mathrm{em}}=751 \mathrm{~nm}$, Table S5 and Fig. S30). Owing to filmformability as well as superior luminescent properties, NIRemissive films, which are promised to be a key material in advanced low-cost and flexible OLEDs, can be readily obtained. In conclusion, robust $\pi$-conjugation systems can be constructed with the boron-fused azomethine units owing to the rigid and planar skeleton. Consequently, NIR emission can be obtained from the bisboron complexes and conjugated polymers. Meanwhile, despite molecular planarity, ACQ can be suppressed in condensed state. According to the X-ray analysis, it is proposed that the substituent at the boron atom could play a critical role in disturbing intermolecular interaction followed by efficient solid-state emission. Our finding might be widely applicable for the design of advanced NIR-emissive materials both in biotechnology and material science.

This work was partially supported by a The Kyoto University Foundation (for M.G.) and a Grant-in-Aid for Young Scientists (for M.G.) (JSPS KAKENHI Grant numbers 18K14275) and Grantin-Aid for ScientificResearch (B) (JSPS KAKENHI Grant numbers 17H03067) and (A) (JSPS KAKENHI Grant numbers 17H01220) and a Grant-in-Aid for Scientific Research on Innovative Areas "New Polymeric Materials Based on Element-Blocks (No.2401)" (JSPS KAKENHI Grant Number 24102013) and a Challenging Research (Pioneering) (JSPS KAKENHI Grant Number 18H05356).

\section{Notes and references}

1 (a) J. Fabian, H. Nakazumi and M. Matsuoka, Chem. Rev., 1992, 92, 1197-1226; (b) G. Yang, J. Liu, Y. Wu, L. Feng and Z. Liu, Coord. Chem. Rev., 2016, 320-321, 100-117; (c) K. Kiyose, H. Kojima and T. Nagano, Chem.-Asian J., 2008, 3, 506-515; (d) Z. Cheng, Y. Wu, Z. Xiong, S. S. Gambhir and X. Chen, Bioconjugate Chem., 2005, 16, 1433-1441.

2 (a) H. Suzuki, A. Yokoo and M. Notomi, Polym. Adv. Technol., 2004, 15, 75-80; (b) N. Tessler, V. Medvedev, M. Kazes, S. H. Kan and U. Banin, Science, 2002, 295, 1506-1508.

3 X. Wang, Z.-Z. Li, S.-F. Li, H. Li, J. Chen, Y. Wu and H. Fu, Adv. Opt. Mater., 2017, 5, 1700027-1700034.

4 (a) A. Zampetti, A. Minotto and F. Cacialli, Adv. Funct. Mater. 2019, 29, 1807623-1807645; (b) D.-H. Kim, A. D'Aléo, X.-K. Chen, A. D. S. Sandanayaka, D. Yao, L. Zhao, T. Komino, E. Zaborova, G. Canard, Y. Tsuchiya, E. Choi, J. W. Wu, F. Fages,
J.-L. Brédas, J.-C. Ribierre and C. Adachi, Nat. Photonics, 2018, 12, 98-104

5 (a) M. Adachi and Y. Nagao, Chem. Mater., 2001, 13, 662669; (b) K. Sezukuri, M. Suzuki, H. Hayashi, D. Kuzuhara, N. Aratani and H. Yamada, Chem. Commun., 2016, 52, 48724875.

6 (a) S. A. Jenekhe, L. Lu and M. M. Alam, Macromolecules, 2001, 34, 7315-7324; (b) H. Van Mullekom, J. Vekemans, E. Havinga and E. Meijer, Mater. Sci. Eng. R, 2001, 32, 1-40; (c) Q. T. Zhang and J. M. Tour, J. Am. Chem. Soc., 1998, 120, 5355-5362; (d) Y. Adachi, Y. Ooyama, Y. Ren, X. Yin, F. Jäkle and J. Ohshita, Polym. Chem., 2018, 9, 291-299.

7 (a) J. Massin, W. Dayoub, J.-C. Mulatier, C. Aronica, Y. Bretonnière and C. Andraud, Chem. Mater., 2011, 23, 862873; (b) X. Cheng, K. Wang, S. Huang, H. Zhang, H. Zhang and Y. Wang, Angew. Chem. Int. Ed., 2015, 54, 8369-8373.

8 (a) R. T. K. Kwok, C. W. T. Leung, J. W. Y. Lam and B. Z. Tang, Chem. Soc. Rev. 2015, 44, 4228-4238; (b) J. Mei, N. L. C. Leung, R. T. K. Kwok, J. W. Y. Lam and B. Z. Tang, Chem. Rev., 2015, 115, 11718-11940.

9 (a) Y. Chujo and K. Tanaka, Bull. Chem. Soc. Jpn., 2015, 88, 633-643; (b) M. Gon, K. Tanaka, and Y. Chujo, Polym. J., 2017, 50, 109-126; (c) A. Lik, L. Fritze, L. Müller and H. Helten, J. Am. Chem. Soc., 2017, 139, 5692-5695; (d) Y. Matsumura, M. Ishidoshiro, Y. Irie, H. Imoto, K. Naka, K. Tanaka, S. Inagi and I. Tomita, Angew. Chem. Int. Ed., 2016, 55, 15040-15043; (e) K. Tanaka and Y. Chujo, Polymer Journal, 2020. https://doi.org/10.1038/s41428-020-0316-y

10 (a) J. S. Dhindsa, R. R. Maar, S. M. Barbon, M. Olivia Avilés, Z. K. Powell, F. Lagugné-Labarthet and J. B. Gilroy, Chem. Commun., 2018, 54, 6899-6902; (b) A. Wakamiya, T. Taniguchi and S. Yamaguchi, Angew. Chem. Int. Ed., 2006, 45 3170-3173; (c) M. Gon, K. Tanaka and Y. Chujo, Angew. Chem. Int. Ed., 2018, 57, 6546-6551; (d) M. Gon, K. Tanaka and Y. Chujo, Bull. Chem. Soc. Jpn., 2019, 92, 7-18.

11 (a) D. Li, H. Zhang and Y. Wang, Chem. Soc. Rev., 2013, 42, 8416-8433; (b) M. Urban, K. Durka, P. Jankowski, J. Serwatowski and S. Luliński, J. Org. Chem., 2017, 82, 82348241; (c) T. Nakamura, S. Furukawa and E. Nakamura, Chem. -Asian J., 2016, 11, 2016-2020; (d) Y. Kubota, K. Kasatani, T. Niwa, H. Sato, K. Funabiki and M. Matsui, Chem. -Eur. J., 2016, 22, 1816-1824; (e) J. Wang, Q. Wu, C. Yu, Y. Wei, X. $\mathrm{Mu}, \mathrm{E}$. Hao and L. Jiao, J. Org. Chem., 2016, 81, 1131611323. (f) Z. Zhang, H. Bi, Y. Zhang, D. Yao, H. Gao, Y. Fan, H. Zhang, Y. Wang, Y. Wang, Z. Chen and D. Ma, Inorg. Chem., 2009, 48, 7230-7236; (g) D. Li, Z. Zhang, S. Zhao, Y. Wang and H. Zhang, Dalton Trans., 2011, 40, 1279-1285; (h) D. Li, K. Wang, S. Huang, S. Qu, X. Liu, Q. Zhu, H. Zhang and Y. Wang, J. Mater. Chem., 2011, 21, 15298-15304. (i) M. Yusuf, K. Liu, F. Guo, R. A. Lalancette and F. Jäkle, Dalton Trans., 2016, 45, 4580-4587. (j) A. Wakamiya, T. Taniguchi and S. Yamaguchi, Angew. Chem. Int. Ed., 2006, 45, 3170-3173. (k) M. J. Calhorda, D. Suresh, P. T. Gomes, R. E. Di Paolo and A. L. Maçanita, Dalton Trans., 2012, 41, 13210-13217.

12 S. Ohtani, M. Gon, K. Tanaka and Y. Chujo, Chem. -Eur. J., 2017, 23, 11827-11833.

13 (a) Y. Dong, J. W. Y. Lam, A. Qin, Z. Li, J. Sun, H. H.-Y. Sung, I. D. Williams and B. Z. Tang, Chem. Commun., 2007, 40-42; (b) P. Galer, R. C. Korošec, M. Vidmar and B. Šket, J. Am. Chem. Soc., 2014, 136, 7383-7394

14 S. Ohtani, M. Gon, K. Tanaka and Y. Chujo, Macromolecules, 2019, 52, 3387-3393.

15 (a) M. Kosugi, K. Sasazawa, Y.Shimizu, T. Migita, Chem. Lett., 1977, 6, 301-302; (b) D. Milstein, J. K. Stille, J. Am. Chem. Soc., 1978, 100, 3636-3638. 